\title{
All-Optical Fiber Interferometer-Based Methods for Ultra-Wideband Signal Generation
}

\author{
Kais Dridi and Habib Hamam \\ EMAT Laboratory, Electrical Department, Faculty of Engineering, University of Moncton, 18 Avenue Antonine Maillet, \\ Moncton, NB, Canada E1A 3E9 \\ Correspondence should be addressed to Kais Dridi, k.dridi@gmail.com and Habib Hamam, habib.hamam@gmail.com \\ Received 28 October 2011; Accepted 8 April 2012 \\ Academic Editor: Baoyong Chi \\ Copyright ( $\odot 2012$ K. Dridi and H. Hamam. This is an open access article distributed under the Creative Commons Attribution \\ License, which permits unrestricted use, distribution, and reproduction in any medium, provided the original work is properly \\ cited.

\begin{abstract}
We report two new, simple, and cost-effective all-optical methods to generate ultra-wideband (UWB) impulse radio signals. The proposed methods are based on fiber-interferometric structures, where an input pulse is split and propagates along the two interferometer arms. The interference of these pulses at the output of the interferometer leads to UWB pulse generation. A theoretical analysis is provided and some relevant simulation results are presented. Large bandwidths are obtained while satisfying the requirements of the Federal Communication Commission (FCC). With these two techniques, UWB pulses can be readily generated and cost-effectively propagated through optical fibers.
\end{abstract}

\section{Introduction}

Major advances in wireless communications, networking, radar, imaging, and positioning systems have been made since the fast emergence of ultra-wideband (UWB) technology. UWB radio technology converges towards being a strongest candidate compared to the other existing radio technologies. It has gained ground since the USA FCC's proposed rulemaking. In its Report and $\operatorname{Order}(\mathrm{R} \& \mathrm{O})$ issued in February 2002, large bandwidth from 3.1 to $10.6 \mathrm{GHz}$ has been unleashed with an isotropic radiated power level of less than $-41 \mathrm{dBm} / \mathrm{MHz}$. Besides, this radio technology owns its success due to its intrinsic features and benefits including low power consumption, low complexity, low cost, wide occupied bandwidth, high data rates, immunity to multipath fading, and high security [1-3].

Unfortunately, shot-range propagation is still considered as a main limitation preventing widespread deployment of such technology: transmitted signals can propagate for distances less than 10 meters as illustrated by Figure 1. To overcome this inherent restriction, solutions based on the integration of optical fibers and UWB radio are promising. The idea is to widen the area of coverage by means of small antennas interconnected by optical fibers. Large surface can hence be divided into small picocells where low-power transmitters are used. A key point in such architecture is the fact that UWB pulses are generated from a central station (CS) and distribute to UWB access points (UWB-AP) through optical fibers as shown in Figure 1. This way saves optoelectronic (O/E) conversions, which limit the bandwidth. In addition, it ensures seamless integration with the high-rate optical networks. Thus, UWB over fiber (UWB-oF) system can be deployed to achieving high data rate access in an indoor environment.

UWB signals have been generated both electronically [4-8] and optically [9-22]. Several approaches have been proposed for optical UWB signal generation. For example, monocycle pulses have been generated using a hybrid system based on a gain switched Fabry-Pérot laser diode (FPLD) and a microwave differentiator $[9,10]$. Other techniques were based on cross-phase modulation (XPM) [11] and cross-gain modulation (XGM) [12] in a semiconductor optical amplifier (SOA). Birefringence time delay has been also applied by cascading phase modulator (PM) with a polarization maintaining fiber (PMF) [13]. Generation based on the gain saturation of a dark return-to-zero (RZ) signal in an SOA 


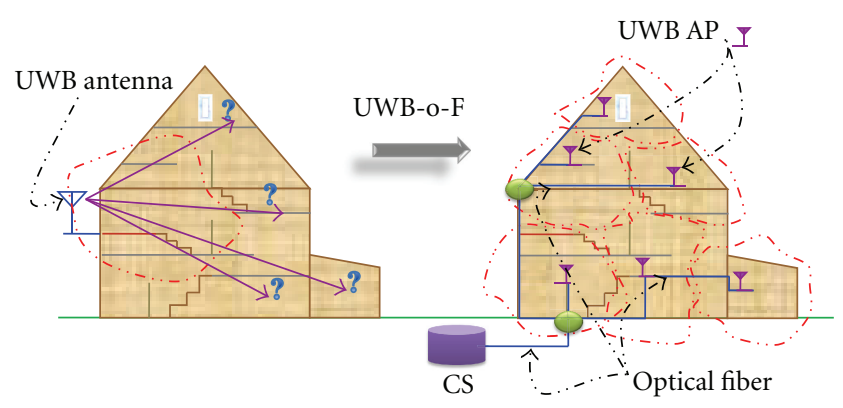

Figure 1: An UWB-o-F system for high data rate coverage (UWBAP: UWB Access Point).

[14] has been also demonstrated. Moreover, other types of UWB signals (e.g., doublet) have been generated based on a Mach-Zehnder modulator (MZM) biased at a nonlinear region [15], a combination of an optical phase modulator with a dispersive fiber [16], and a special frequency shift keying modulator [17], and a conversion from phase modulation (PM) to intensity modulation (IM) is performed by an optical frequency discriminator-based Fiber Bragg Grating (FBG) [18-22]. The aforementioned techniques require a lot of adjustment and control to be able to ensure a relative stable UWB pulse generation. This may lead to complex solutions, which will be costly when implementing. In this paper, we advance two new and simple approaches for monocycle and doublet UWB signal generation in the optical domain.

\section{Development of the Design Methods}

In nonlinear (NL) dispersive fibers, propagation of optical pulses is governed by a propagation equation which can be reduced to the nonlinear Schrödinger Equation (NLSE) under certain conditions [23]. The propagation equation can be written as follows:

$$
i \frac{\partial u}{\partial z}+i \beta_{1} \frac{\partial u}{\partial t}+i \frac{\alpha}{2} u-\frac{1}{2} \beta_{2} \frac{\partial^{2} u}{\partial t^{2}}-\frac{i}{6} \beta_{3} \frac{\partial^{3} u}{\partial t^{3}}+\gamma|u|^{2} u=0,
$$

where $u$ is the slowly varying amplitude of the incident pulse envelope, $\alpha$ is the attenuation coefficient related to fiber losses effects, and $\beta_{2}$ and $\beta_{3}$ are, respectively, the secondand third-order dispersion parameters: the group velocity dispersion (GVD) $\left(\left[\mathrm{ps}^{2} / \mathrm{Km}\right]\right)$ and the third-order dispersion (TOD) $\left(\left[\mathrm{ps}^{3} / \mathrm{Km}\right]\right)$ parameters. The nonlinear parameter is represented by $\gamma$ known as the self-phase modulation (SPM) parameter. $\beta_{1}$ is the first-order dispersion constant or merely the inverse of the group velocity $v_{g}([\mathrm{ps} / \mathrm{Km}])$.

Suppose that a related time frame $T$ which is measured in a frame of reference moving with the pulse at the group velocity $v_{g}\left(T=\tau-\left(\mathrm{z} / v_{\mathrm{g}}\right)=\mathrm{t}-\beta_{1} \mathrm{z}\right)$. The $\beta_{1}$ term of $(1)$ can be omitted. Besides, since pulses are significantly larger than 5 picoseconds, TOD as well as nonlinear effects can also be neglected [24]. In a previous work and for another application a more rigorous analysis including TOD was carried out [24]. However in our present application the pulse width is larger than $10 \mathrm{ps}$, which means that TOD and nonlinear effects have insignificant meaning. By neglecting the TOD (and preserving the non linear parameter $\gamma$ for the moment) we obtain

$$
i \frac{\partial u}{\partial z}+i \frac{\alpha}{2} u-\frac{1}{2} \beta_{2} \frac{\partial^{2} u}{\partial T^{2}}+\gamma|u|^{2} u=0 .
$$

Let us consider the case where only the dispersion is dominating and the pulse is propagating in a lossless medium $(\alpha=0)$; (2) can be rewritten as

$$
i \frac{\partial u}{\partial z}=\frac{1}{2} \beta_{2} \frac{\partial^{2} u}{\partial T^{2}} .
$$

Nonlinearity effects can be neglected under the following condition: $L_{d} / L_{n l} \ll 1$, where $L_{d}$ is the dispersion length expressed by $T_{0}^{2} /\left|\beta_{2}\right|$ and $L_{n l}$ is the nonlinear length expressed by $1 / \gamma_{P_{0}} . P_{0}$ and $T_{0}$ are, respectively, the peak power and initial width for the incident pulse. For given values of the fiber parameters $\gamma$ and $\beta_{2}, P_{0}$ should be $\ll 1$. $\mathrm{W}$ and $T_{0}$ should be in the picoseconds range.

Consider now a normalized amplitude $s(z, \tau)$ introduced as follows:

$$
u(z, \tau)=\sqrt{P_{0}} \exp \left(-\frac{\alpha z}{2}\right) s(z, \tau)
$$

where $\tau$ is a time scale-normalized to the input pulse width $T_{0}$ - defined by $T / T_{0}$.

Under all the aforementioned conditions, if we define the normalized amplitude $s(z, T)$ according to $(4), s(z, T)$ satisfies the following partial differential equation:

$$
i \frac{\partial s}{\partial z}=\frac{1}{2} \beta_{2} \frac{\partial^{2} s}{\partial T^{2}} .
$$

The expansion of (5) leads to

$$
i(s(z+\Delta z, T)-s(z, T))=\frac{1}{2} \Delta z \beta_{2} \frac{\partial^{2} s}{\partial T^{2}} .
$$

Let us suppose the difference between the two fields $s(z+$ $\Delta z, T)$ and $s(z, T)$ as an output signal described by the following expression:

$$
s^{1}{ }_{\text {out }}(z, T)=s(z+\Delta z, T)-s(z, T) .
$$

Expression (7) represents a spatial difference since it is a difference fulfilled in the space (i.e., $z$ ) domain. In addition to this spatial difference, a temporal difference can be achieved as well. This is done by fixing $z$ and varying $T$ by a certain delay of $\tau$. Hence, another difference results as

$$
s^{2} \text { out }(z, T)=s(z, T-\tau)-s(z, T) .
$$

A Fourier domain analysis tells us that

$$
\begin{aligned}
& T F\left(s^{1}{ }_{\text {out }}(z, T)\right) \alpha-\omega^{2} S(z, \omega), \\
& T F\left(s^{2}{ }_{\text {out }}(z, T)\right) \alpha-\omega S(z, \omega) .
\end{aligned}
$$




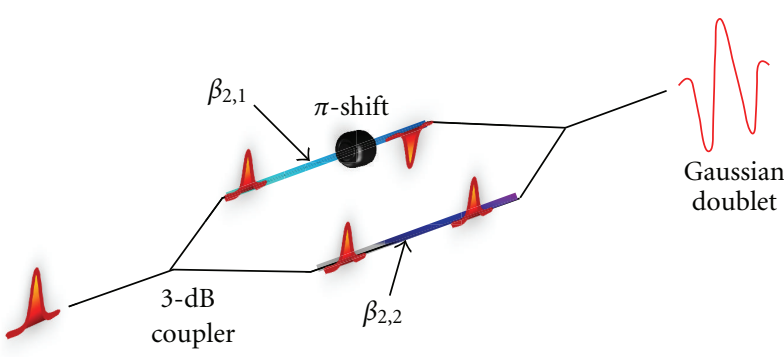

Figure 2: Possible doublet pulse generation scheme.

Going back to time domain, $s^{1}$ out and $s^{2}$ out are proportional to the first and second derivatives of the impulse $s$ itself:

$$
\begin{aligned}
& s^{1}{ }_{\text {out }}(z, T) \alpha s^{(2)}(z, T), \\
& s^{2}{ }_{\text {out }}(z, T) \alpha s^{(1)}(z, T) .
\end{aligned}
$$

Two possible schemes can hence be proposed for the generation of UWB signals. In both cases the first and the second derivates of a Gaussian impulse give the monocycle and the doublet pulses, respectively $[25,26]$.

2.1. Space-Based UWB Pulse Generation. Figure 2 shows us a clear insight on how a physical implementation for UWB impulses generation would be: an interferometer system having two arms with different lengths $z$ and $z+\Delta z$. The $\pi$ shift element induces a negative impulse.

In the following analysis, we used the expressions of $s_{1}$ and $s_{2}$ that can be deduced from the solution of (5) in two different ways:

$$
\begin{aligned}
s_{1}(z, T)= & s(z, T) \\
= & \frac{T_{0}}{\left(T_{0}-i \beta_{2,1} z\right)^{1 / 2}} \exp \left(-\frac{T^{2}}{2\left(T_{0}^{2}-i \beta_{2,1} z\right)}\right), \\
s_{2}(z, T)= & s(z+\Delta z, T) \\
= & \frac{T_{0}}{\left(T_{0}-i \beta_{2,1}(z+\Delta z)\right)^{1 / 2}} \\
& \times \exp \left(-\frac{T^{2}}{2\left(T_{0}^{2}-i \beta_{2,1}(z+\Delta z)\right)}\right) .
\end{aligned}
$$

$\beta_{2}$ in (5) is $\beta_{2,1}$.

Equation (12) contains a virtual GVD parameter $\beta_{2,2}$, which is different from the first one $\left(\beta_{2,1}\right)$ :

$$
\beta_{2,2}=\beta_{2,1} \frac{z+\Delta z}{z}
$$

So, instead of adding a short fiber-length $(\Delta z)$ to the second arm, we can choose a fiber arm as long as the first arm but with a different dispersion parameter as depicted in Figure 3.

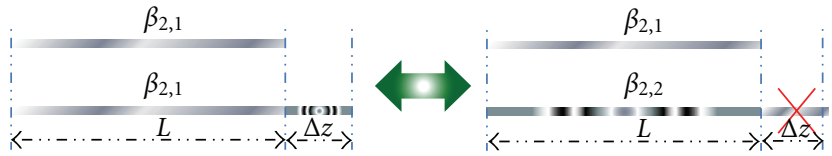

Figure 3: Length-dispersion equivalence.

$$
\text { Expression (12) becomes }
$$

$$
s_{2}(z, T)=s(z+\Delta z, T)
$$

$$
=\frac{T_{0}}{\left(T_{0}-i \beta_{2,2} z\right)^{1 / 2}} \exp \left(-\frac{T^{2}}{2\left(T_{0}^{2}-i \beta_{2,2} z\right)}\right) .
$$

For a fixed first arm length $z=L, \beta_{2,2}$ is constant. As a special case, if we fix $z$ to $L_{d}$, which is the dispersion length over which the effects of dispersion become more important (introduced in Section 2), the following relationship will have an important consideration in our simulation:

$$
\beta_{2,2}=\beta_{2,1} \frac{L+\Delta z}{L} .
$$

2.2. Time-Delayed UWB Generation. Figure 4 depicts another possible interferometer-based system, which is composed of two optical fiber arms with different lengths, two optical 3 - $\mathrm{dB}$ couplers, and a $\pi$-phase shifting device. The delay loop element assures a delay $\tau$ between the two arms. Fourier transform of the expression (8) leads to

$$
S_{\text {out }}(z, \omega)=\left(e^{-i \omega \tau}-1\right) S(z, \omega),
$$

where $S$ and $S_{\text {out }}$ are, respectively, the Fourier transforms of both the injected and the output pulses. The decomposition of (16) into Taylor basis would lead to (up to the first order)

$$
S_{\text {out }}(z, \omega) \approx-i \omega \tau S(z, \omega) .
$$

From (17) we can make out that the output spectrum is identical to the initial spectrum modulated by a linear function of the frequency. Let us replace $S(z, \omega)$ by the following analytical expression:

$$
S(z, \omega)=S(0, \omega) \exp \left(i \frac{\beta_{2}}{2} \omega^{2} z\right),
$$

where $S(0, \omega)$ can be calculated as [18]

$$
S(0, \omega)=\sqrt{2 \pi} T_{0} \exp \left(-\frac{\omega^{2} T_{0}{ }^{2}}{2}\right) .
$$

Hence, by combining (18) and (19), the spectrum of the output signal becomes

$$
\begin{aligned}
S_{\text {out }}(z, \omega)= & \sqrt{2 \pi} T_{0}\left(e^{-i \omega \tau}-1\right) \exp \left(i \frac{\beta_{2}}{2} \omega^{2} z\right) \\
& \times \exp \left(-\frac{\omega^{2} T_{0}^{2}}{2}\right) .
\end{aligned}
$$


TABLE 1: Doublet generation's parameters.

\begin{tabular}{lccccccc}
\hline$P_{0}$ & FWHM & CR1 & CR2 & $l_{1}$ & $l_{2}$ & $\beta_{2,1}$ & $\beta_{2,2}$ \\
\hline $0.95 \mathrm{~mW}$ & $62.5 \mathrm{ps}$ & 0.3 & 0.1 & $720 \mathrm{~m}$ & $720 \mathrm{~m}$ & $-7.34 \mathrm{ps}^{2} / \mathrm{km}^{2}$ & $-5.75 \mathrm{ps}^{2} / \mathrm{km}^{2}$ \\
\hline
\end{tabular}

TABLE 2: Monocycle generation's parameters.

\begin{tabular}{lccccccc}
\hline$P_{0}$ & FWHM & CR1 & CR2 & $l_{1}$ & $l_{2}$ & $\beta_{2,1}$ & $\beta_{2,2}$ \\
\hline $2 \mathrm{~mW}$ & $46.875 \mathrm{ps}$ & 0.5 & 0.5 & $720 \mathrm{~m}$ & $500 \mathrm{~m}$ & $-22.28 \mathrm{ps}^{2} / \mathrm{km}^{2}$ & $-22.28 \mathrm{ps}^{2} / \mathrm{km}^{2}$ \\
\hline
\end{tabular}

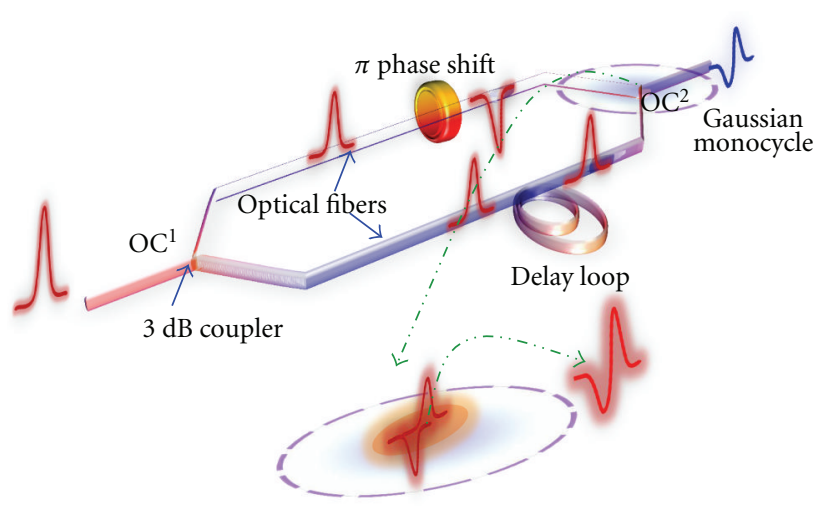

Figure 4: Time delay interferometer (OC: Optical Coupler).

Since the initial spectrum $S(0, \omega)$ is as large as that of the propagated signal $S(z, \omega)$, the bandwidth of the output signal (the extent of $S_{\text {out }}(z, \omega)$ ) does not depend on $z$.

In the two proposed systems, a $\pi$ phase device must be inserted in one arm to implement the negative impulse function. The $\pi$-phase shift element design is not considered in our work; however, it can be implemented such as in [27] or [28].

\section{Simulation Results}

As a quick proof of concept, we have carried out simulations using the Photonic Transmission Design System (PTDS) simulator produced by Virtual Photonics Inc, known as VPISystems nowadays [29]. It is based on the Ptolemy opensource software [30]. Figure 5 describes a range of parameters that have been manipulated in the purpose of ensuring high-quality monocycle and doublet impulses. The injected impulse is characterized by its initial power $\left(P_{0}\right)$ and its full width at half maximum (FWHM). The optical couplers control the amount of power through the interferometer by adjusting the coupling ratios CR1 and CR2. $l_{1}, l_{2}, \beta_{2,1}$, and $\beta_{2,2}$ are the lengths and GVD parameters of the two optical fiber arms, respectively. The semiconductor optical amplifier (SOA) gives a little amplification for the obtained impulse. In these simulations, it has been controlled through its injection current $I_{c}$. A photodetector performs a conversion from the optical to the electrical domain.

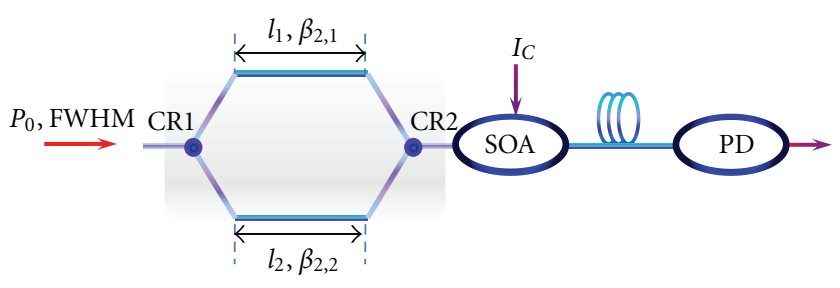

FIgURE 5: UWB pulse generation schema.

3.1. For the Space Approach. In this approach, the interferometer arms' lengths are chosen equal to $720 \mathrm{~m}$ (Figure 5) but with different dispersion parameters $-7.34 \mathrm{ps}^{2} / \mathrm{km}$ and $-5.75 \mathrm{ps}^{2} / \mathrm{km}$. A Gaussian pulse, with an FHWM of $62.5 \mathrm{ps,}$ is injected via the first optical coupler which has a coupling ration CR1 equal to 0.3. A Gaussian doublet has been generated and amplified with an SOA (biased at $150 \mathrm{~mA}$ ). Table 1 summarizes the interferometer's parameters used for the doublet generation. A UWB doublet signal has been obtained with an FWHM of about 48.45 ps, as shown in Figure 6, with $10.7 \mathrm{GHz}$ bandwidth (from 2.7 to $13.4 \mathrm{GHz}$ ) measured at $-10 \mathrm{~dB}$.

3.2. For the Time-Delay Approach. A $2 \mathrm{~mW}$ Gaussian pulse is launched to the interferometer system with an FWHM of about $47 \mathrm{ps}$ (Figure 5). The arms' lengths are fixed to $720 \mathrm{~m}$ and $520 \mathrm{~m}$ with the same dispersion parameter of $-22 \mathrm{ps}^{2} / \mathrm{km}$. The coupling ratios of the input and output couplers of our system are equal to 0.5 (See Table 2 for the interferometer's parameters). At the output of the second coupler, the optical monocycle pulse is amplified by a semiconductor optical amplifier (SOA) biased at $120 \mathrm{~mA}$. Table 2 summarized the interferometer's parameters used for monocycle generation. After the photodetector, the resulted monocycle (Figure 7) measures an upper FWHM of about 40.625 ps and a lower FWHM of about 57.813 ps. It offers a $-10 \mathrm{~dB}$ bandwidth of about $10.3 \mathrm{GHz}$ from 2.3 to $12.6 \mathrm{GHz}$ (Figure 7 ). The obtained spectrum respects well the requirements of the FCC spectral mask.

Both symmetry of the monocycle and the bandwidth of its spectrum can be adjusted by tuning the injection current in the SOA. The higher this current is, the more the symmetric pulse is ensured.

The time-delay approach offers a relatively broader spectrum than that obtained with the space approach. On the other hand, from a practical point of view, the latter 

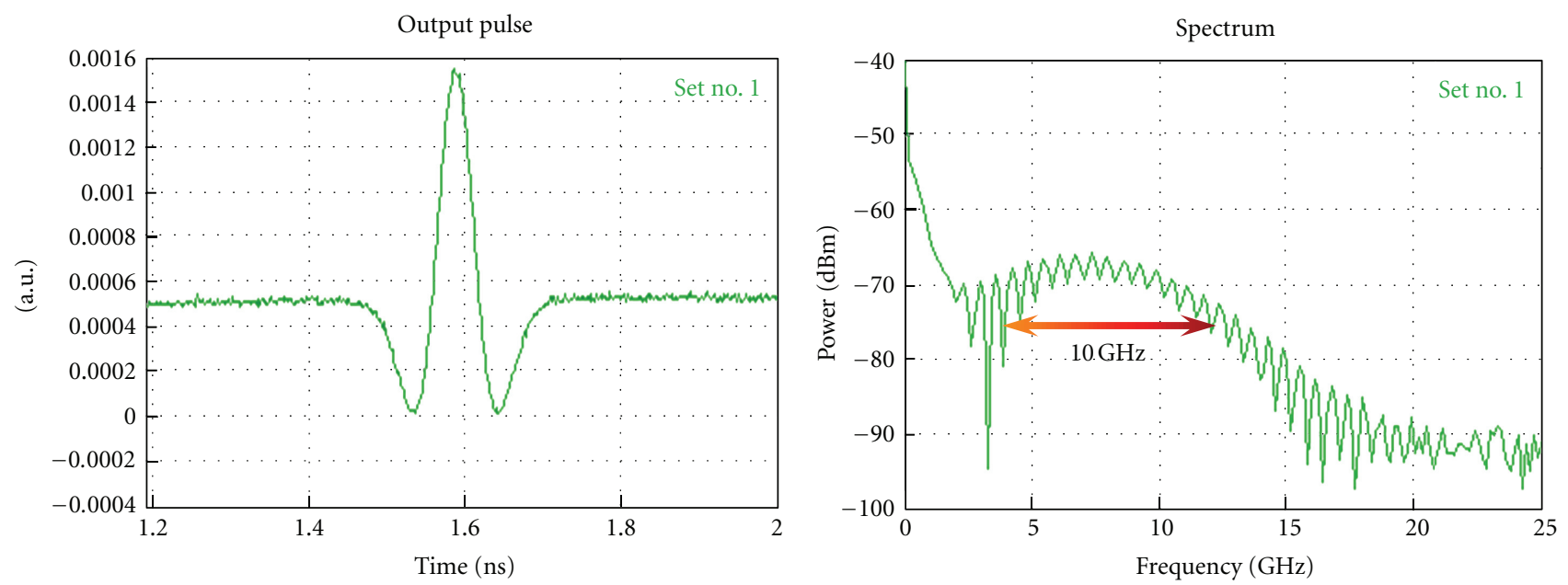

Figure 6: The generated doublet (a) and its spectrum (b).
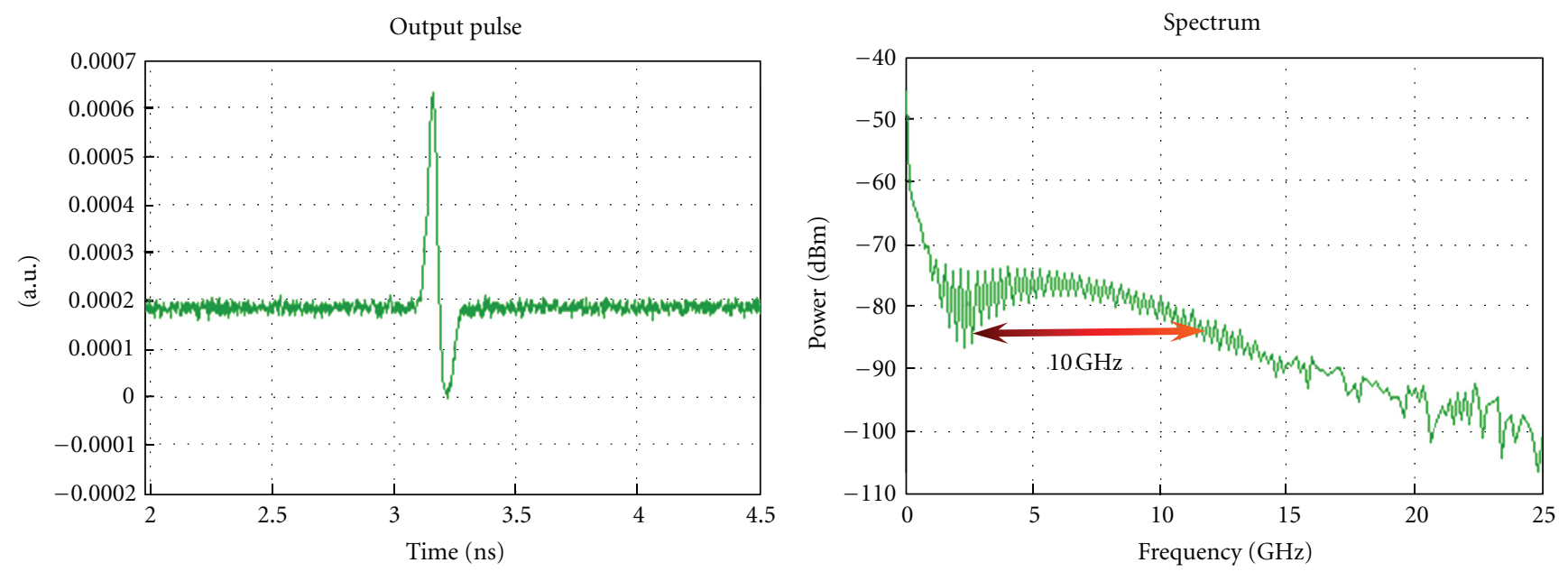

Figure 7: The generated monocycle (a) and its spectrum (b).

approach not only requires different and nonstandard dispersion parameters in both arms of the proposed system, but also imposes the use of fiber optic couplers with specific coupling ratios. Conversely, the former approach requires only standard fiber arms (e.g., a standard Corning SMF-28) with $50 \%$ fiber optic couplers. The time-delay approach is thus more simple and cost-effective solution for experimental investigation, albeit it shows comparative results with the other method.

\section{Conclusion}

New all-optical UWB pulses generation methods have been demonstrated and approved by simulations. While the first one is based on time-delay approach to generate monocycle pulse, the second approach has a spatial perspective where the chromatic dispersion is exploited to generate doublet pulse. Both methods use an interferometric architecture incorporating a $\pi$-device shift. Interesting bandwidths have been obtained meeting the FCC requirements. With these methods, not only can UWB pulses be generated optically but also their propagation over optical networks is simply assured. Experimental assessment would be a key point as a future work.

\section{References}

[1] G. R. Aiello, "Challenges for Ultra-wideband (UWB) CMOS integration," in Proceedings of the IEEE MTT-S International Microwave Symposium Digest, vol. 1, pp. 361-364, June 2003.

[2] D. Porcino and W. Hirt, "Ultra-wideband radio technology: potential and challenges ahead," IEEE Communications Magazine, vol. 41, no. 7, pp. 66-74, 2003.

[3] M. Ghavami, L. B. Michael, and R. Kohno, Ultra WideBand Signals and Systems in Communication Engineering, Wiley, West Sussex, UK, 2004.

[4] X. Chen and S. Kiaei, "Monocycle shapes for ultra wideband system," in Proceedings of the IEEE International Symposium on Circuits and Systems, vol. 1, pp. 26-29, May 2002. 
[5] K. Marsden, H.-J. Lee, D. S. Ha, and H.-S. Lee, "Low power CMOS re-programmable pulse generator for UWB systems," in Proceedings of the IEEE Conference on Ultra Wideband Systems and Technologies, pp. 337-443, November 2003.

[6] H. Kim, D. Park, and Y. Joo, "All-digital low-power CMOS pulse generator for UWB system," Electronics Letters, vol. 40, no. 24, pp. 1534-1535, 2004.

[7] Y. Jeong, S. Jung, and J. Liu, "A CMOS impulse generator for UWB wireless communication systems," in Proceedings of the IEEE International Symposium on Cirquits and Systems, pp. 129-132, May 2004.

[8] B. Jung, Y. H. Tseng, J. Harvey, and R. Harjani, "Pulse generator design for UWB IR communication systems," in Proceedings of the IEEE International Symposium on Circuits and Systems (ISCAS '05), pp. 4381-4384, May 2005.

[9] W. P. Lin and J. Y. Chen, "Implementation of a new ultrawideband impulse system," IEEE Photonics Technology Letters, vol. 17, no. 11, pp. 2418-2420, 2005.

[10] W. P. Lin and Y. C. Chen, "Design of a new optical impulse radio system for ultra-wideband wireless communications," IEEE Journal on Selected Topics in Quantum Electronics, vol. 12 , no. 4 , pp. 882-887, 2006.

[11] J. Dong, X. Zhang, J. Xu, D. Huang, S. Fu, and P. Shum, "Ultrawideband monocycle generation using cross-phase modulation in a semiconductor optical amplifier," Optics Letters, vol. 32, no. 10, pp. 1223-1225, 2007.

[12] Q. Wang, F. Zeng, S. Blais, and J. Yao, "Optical ultrawideband monocycle pulse generation based on cross-gain modulation in a semiconductor optical amplifier," Optics Letters, vol. 31, no. 21, pp. 3083-3085, 2006.

[13] H. Chen, M. Chen, C. Qiu, J. Zhang, and S. Xie, "UWB monocycle pulse generation by optical polarisation time delay method," Electronics Letters, vol. 43, no. 9, pp. 542-543, 2007.

[14] J. Dong, X. Zhang, J. Xu, and D. Huang, "All-optical ultrawideband monocycle generation utilizing gain saturation of a dark return-to-zero signal in a semiconductor optical amplifier," Optics Letters, vol. 32, no. 15, pp. 2158-2160, 2007.

[15] Q. Wang and J. Yao, "UWB doublet generation using nonlinearly-biased electro-optic intensity modulator," Electronics Letters, vol. 42, no. 22, pp. 1304-1305, 2006.

[16] F. Zeng and J. Yao, "An approach to ultrawideband pulse generation and distribution over optical fiber," IEEE Photonics Technology Letters, vol. 18, no. 7, pp. 823-825, 2006.

[17] T. Kawanishi, T. Sakamoto, and M. Izutsu, "Ultra-wide-band radio signal generation using optical frequency-shift- keying technique," IEEE Microwave and Wireless Components Letters, vol. 15, no. 3, pp. 153-155, 2005.

[18] F. Zeng and J. Yao, "Optical generation and distribution of UWB signals," in Proceedings of the International Conference on Communications, Circuits and Systems (ICCCAS '06), vol. 3, pp. 2024-2029, June 2006.

[19] F. Zeng and J. Yao, "Ultrawideband impulse radio signal generation using a high-speed electrooptic phase modulator and a fiber-bragg-grating-based frequency discriminator," IEEE Photonics Technology Letters, vol. 18, no. 19, pp. 2062-2064, 2006.

[20] F. Zeng, Q. Wang, and J. Yao, “All-optical UWB impulse generation based on cross-phase modulation and frequency discrimination," Electronics Letters, vol. 43, no. 2, pp. 119-121, 2007.

[21] C. Wang, F. Zeng, and J. Yao, "All-fiber ultrawideband pulse generation based on spectral shaping and dispersion-induced frequency-to-time conversion," IEEE Photonics Technology Letters, vol. 19, no. 3, pp. 137-139, 2007.
[22] J. Li, K. Xu, S. Fu et al., "Ultra-wideband pulse generation with flexible pulse shape and polarity control using a Sagnacinterferometer-based intensity modulator," Optics Express, vol. 15, no. 26, pp. 18156-18161, 2007.

[23] G. P. Agrawal, Nonlinear Fiber Optics, Academic Press, 3rd edition, 2001.

[24] M. Razzak, S. Guizani, H. Hamam, and Y. Bouslimani, "Optical post-equalization based on self-imaging," Journal of Modern Optics, vol. 53, no. 12, pp. 1675-1684, 2006.

[25] X. Chen and S. Kiaei, "Monocycle shapes for ultra wideband system," in Proceedings of the IEEE International Symposium on Circuits and Systems, pp. 597-600, May 2002.

[26] B. Hu and N. C. Beaulieu, "Pulse shapes for ultrawideband communication systems," IEEE Transactions on Wireless Communications, vol. 4, no. 4, pp. 1789-1797, 2005.

[27] J. R. Kurz, K. R. Parameswaran, R. V. Roussev, and M. M. Fejer, "Optical-frequency balanced mixer," Optics Letters, vol. 26, no. 16, pp. 1283-1285, 2001.

[28] O. Deparis, R. Kiyan, O. Pottiez et al., "Bandpass filters based on $\pi$-shifted long-period fiber gratings for actively mode-locked erbium fiber lasers," Optics Letters, vol. 26, no. 16, pp. 12391241, 2001.

[29] VPISystems, http://www.vpisystems.com/.

[30] Ptolemy Project, http://www.ptolemy.eecs.berkeley.edu/index .htm. 

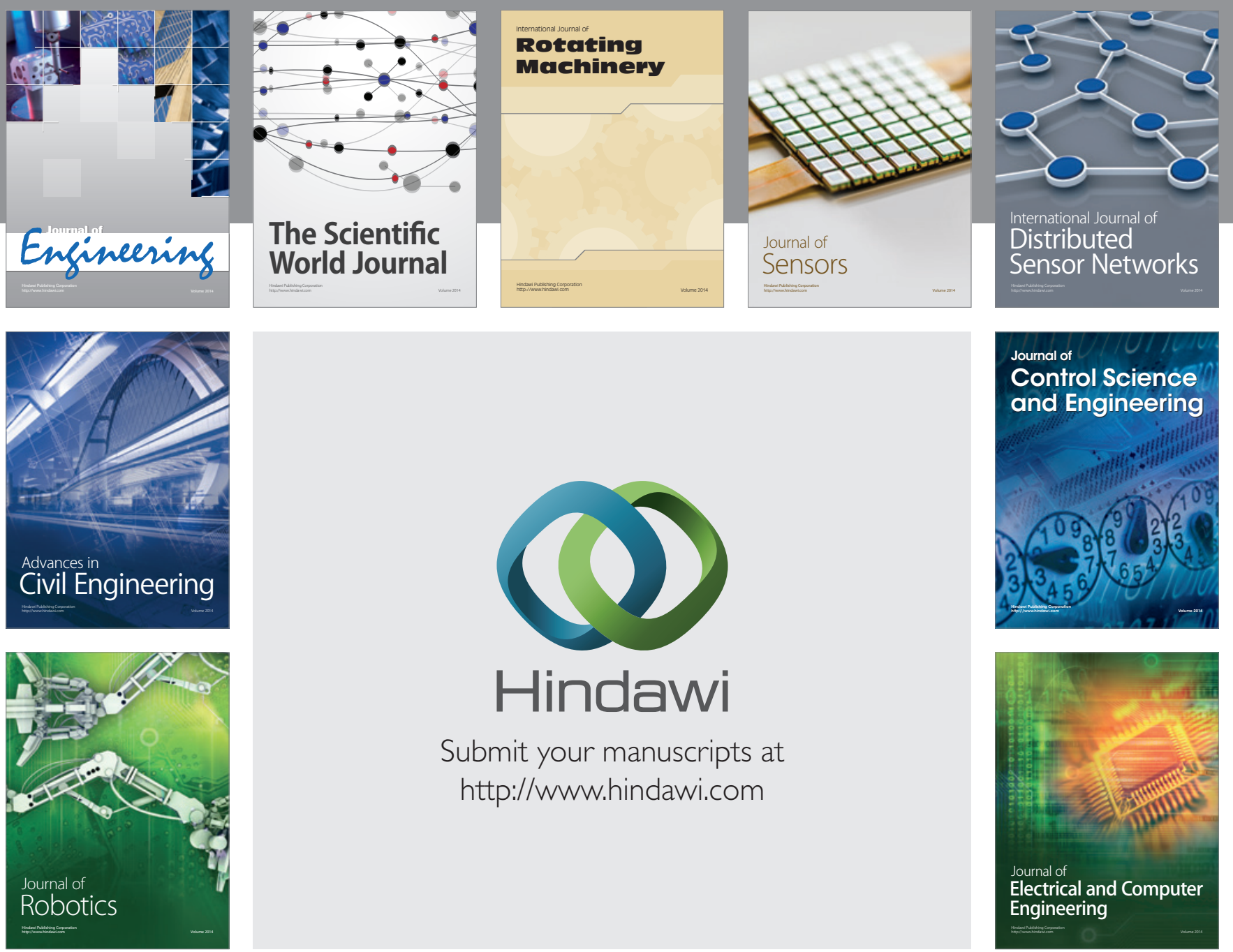

Submit your manuscripts at

http://www.hindawi.com
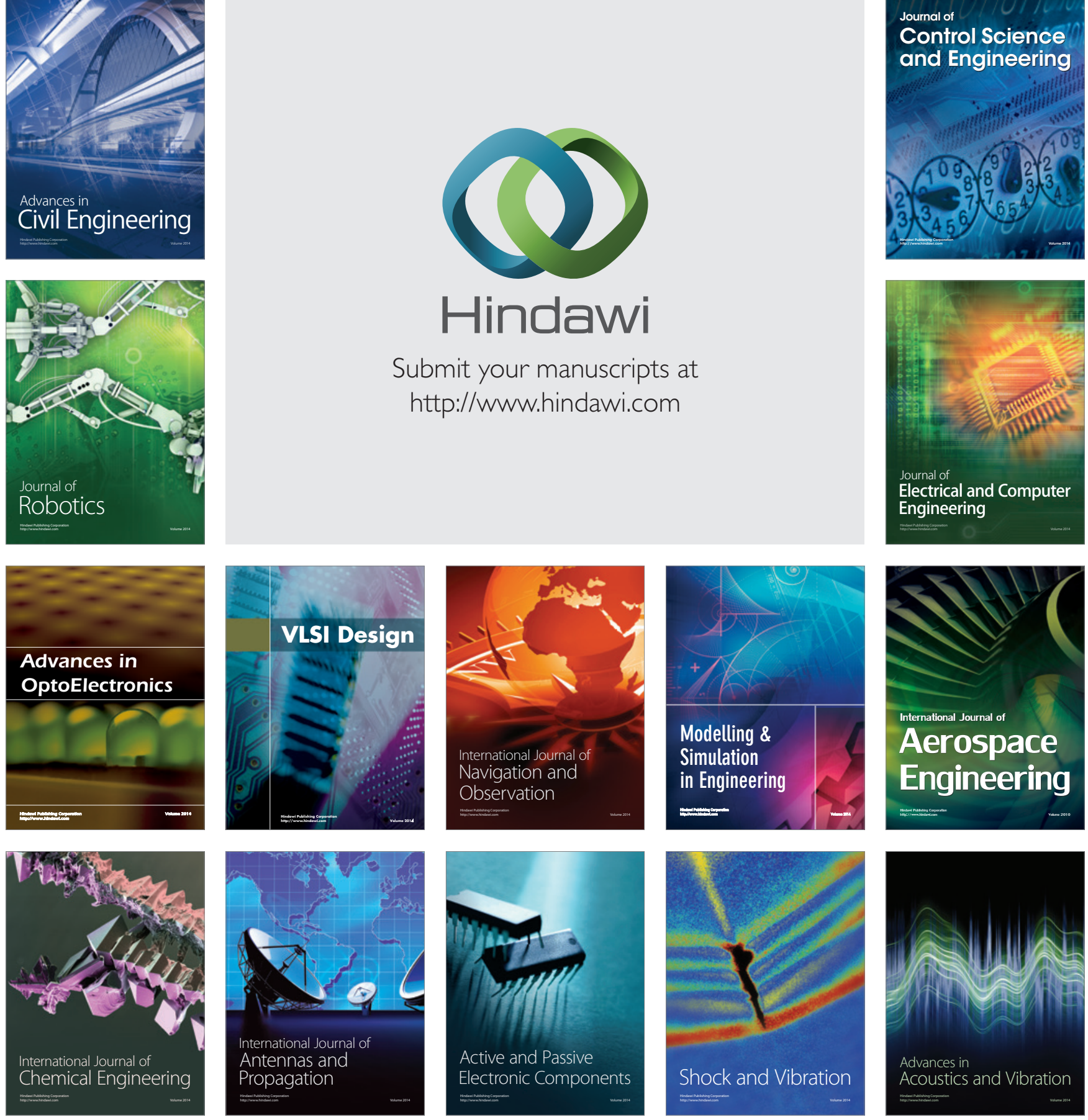